\title{
KÄHLER METRIC ON THE SPACE OF CONVEX REAL PROJECTIVE STRUCTURES ON SURFACE
}

\author{
INKANG KIM AND GENKAI ZHANG
}

\begin{abstract}
We prove that the space of convex real projective structures on a surface of genus $g \geq 2$ admits a mapping class group invariant Kähler metric where Teichmüller space with Weil-Petersson metric is a totally geodesic complex submanifold.
\end{abstract}

\section{INTRODUCTION}

Recently, the character variety $\chi\left(\pi_{1}(M), G\right)$ of representations of $\pi_{1}(M)$ in a real algebraic group $G$, has drawn many attentions from different branches of mathematics. The $G$-character variety $\chi\left(\pi_{1}(M), G\right)$ is the geometric quotient of $\operatorname{Hom}\left(\pi_{1}(M), G\right)$ by inner automorphisms of $G$. Often, some components of the character variety correspond to some geometric structures on $M$. Hitchin [16] introduced Hitchin component in the character variety of a closed surface group in $P S L(n+1, \mathbb{R})$ generalizing Teichmüller space in $\operatorname{PSL}(2, \mathbb{R})$. More precisely, he showed that for the adjoint group $G$ of the split real form of a complex simple Lie group $G^{c}$, the quotient by the conjugation action of $G$ of the set of homomorphisms, from the fundamental group $\Gamma$ of a closed surface $S$ of genus $g \geq 2$ to $G$, which acts completely reducibly on the Lie algebra of $G$, has a connected component homeomorphic to Euclidean space of dimension $(2 g-2) \operatorname{dim} G$. His method is the use of Higgs bundle theory developed by himself, K. Corlette, S. Donaldson, C. Simpson and many others [15, 31]. A homomorphism from $\Gamma$ to $G^{c}$ defines a flat principal $G^{c}$-bundle. Given a complex structure on $S$, denoted by $\Sigma$, a theorem of Corlette and Donaldson associates a natural $G^{u}$-connection $A$, where $G^{u}$ is the maximal compact subgroup of $G^{c}$, and a Higgs field $\Psi \in H^{0}(\Sigma, \operatorname{ad} P \otimes \mathcal{K})$ which satisfy the equation $F_{A}+\left[\Psi, \Psi^{*}\right]=0$. Here $\mathcal{K}$ is the canonical line bundle over $\Sigma, P$ is a principal $G^{u}$ bundle and $\operatorname{ad} P$ is the Lie algebra bundle associated to the adjoint representation of $G^{u}$. Solutions to these equations provide a holomorphic parametrization of the equivalence classes of homomorphisms from $\Gamma$ to $G^{c}$. For appropriately chosen $\Psi$ the solutions are stable under the complex conjugation in $G^{c}$ and reduces to $G$-connection corresponding to elements in the Hitchin component.

\footnotetext{
${ }^{1} 2000$ Mathematics Subject Classification. 51M10, 57S25.

${ }^{2}$ Key words and phrases. Hitchin component, real projective structure, Kähler metric.

${ }^{3}$ Research partially supported by STINT-NRF grant (2011-0031291). Research by G. Zhang is supported partially by the Swedish Science Council (VR). I. Kim gratefully acknowledges the partial support of grant (NRF-2014R1A2A2A01005574) and a warm support of Chalmers University of Technology during his stay.
} 
For the real linear group $S L(n+1, \mathbb{R})$, Hitchin showed that the Hitchin component is homeomorphic to $\bigoplus_{j=1}^{n} H^{0}\left(\Sigma, \mathcal{K}^{j+1}\right)$. We need to mention that this homeomorphism depends on a priori fixed complex structure on $S$, and hence it is not mapping class group equivariant. After Hitchin's work, many people pursued to clarify this component in many different ways. Notably Labourie [21] introduced a notion of Anosov representations and proved that Hitchin representations are exactly Anosov representations in $S L(n+1, \mathbb{R})$. In [22], he also suggested a mapping class group equivariant parametrization using Hitchin map and an adaptation of an energy functional over Teichmüller space. We will review his interpretation of Hitchin map in Section 3 .

It has been conjectured for a long time that the Hitchin component admits a mapping class group invariant Kähler metric. There have been many evidences for this, see [13, 24, 6]. In the last section, we prove the existence of a mapping class group invariant Kähler metric on the Hitchin component for $n=2$.

Theorem 1.1. The Hitchin component of the character variety $\chi\left(\pi_{1}(M), S L(3, \mathbb{R})\right)$ can be equipped with a mapping class group invariant Kähler metric where $M$ is a closed surface of genus $\geq 2$. Furthermore Teichmüller space equipped with the Weil-Petersson metric is a totally geodesic complex submanifold.

This Kähler metric is constructed using certain $L^{2}$-metric. Intuitively, we glue WeilPetersson metric on the base and $L^{2}$-metric along vertical fibers using Griffith negativity. Indeed, we need the dual of a holomorphic vector bundle over Teichmüller space whose fibres are cubic holomorphic forms. The geometric properties of this metric such as various curvatures, geodesics will be explored in a near future. We hope that this new natural Kähler metric will help us to better understand the moduli space of real projective structures and the Teichmüller space as a byproduct.

This particular Hitchin component has been intensively studied by many people. ChoiGoldman showed that the corresponding geometric structure is the convex real projective structure [7] and the bundle structure is verified by Labourie [20] and Loftin [27] independently using Monge-Àmpere equations relying on the seminal work of Cheng-Yau [8]. The symplectic structure on the Hitchin component has been studied by Goldman [13]. Recently Li [24] constructed a mapping class group invariant metric using explicit constructions over $\mathfrak{s l}(3, \mathbb{R})$-bundles and Cheng-Yau metric over the cone. It would be interesting to compare this construction with ours. Bridgeman-Canary-Labourie-Sambarino [6] constructed a pressure metric on Hitchin component of $S L(n+1, \mathbb{R})$ for every $n$ using different method.

Acknowledgement We would like to thank Bo Berndtsson for a few helpful discussions on complex vector bundles, in particular for Kähler property based on Griffith's positivity. We are grateful for the anonymous referee for valuable suggestions and for the careful reading of an earlier version of this paper. 


\section{Convex Projective Structures on A MANifold $M$}

A flat projective structure on an $n$-dimensional manifold $M$ is a $\left(\mathbb{R P}^{n}, P S L(n+1, \mathbb{R})\right)$ structure, i.e., there exists a maximal atlas on $M$ whose transition maps are restrictions to open sets in $\mathbb{R P}^{n}$ of elements in $P S L(n+1, \mathbb{R})$. Then there exist a natural holonomy map $\rho: \pi_{1}(M) \rightarrow P S L(n+1, \mathbb{R})$ and a developing map from the universal cover $\tilde{M}$, $f: \tilde{M} \rightarrow \mathbb{R P}^{n}$ such that

$$
\forall x \in \tilde{M}, \forall \gamma \in \pi_{1}(M), f(\gamma x)=\rho(\gamma) f(x) .
$$

We will consider projective structures deformed from hyperbolic structures, and all holonomy representations will lift to $S L(n+1, \mathbb{R})$. An $\mathbb{R} \mathbb{P}^{n}$-structure is convex if the developing map is a homeomorphism onto a convex domain in $\mathbb{R} \mathbb{P}^{n}$. It is properly convex if the domain is included in a compact convex set of an affine chart, strictly convex if the convex set is strictly convex.

When $M=S$ is a closed Riemann surface of genus at least 2, a huge amount of literature for the set of marked strictly convex real projective structures on $S$ exist concerning its parametrization [14], its identification with Hitchin component [7], degeneration of the projective structures [18], entropy of geodesic flow [12], the marked length rigidity [17], its Zariski tangent space at Fuchsian locus of the character variety in $S L(n, \mathbb{R})$ [19] and many more. For a recent generalization to finite volume convex real projective structures, see [3, 10].

In this paper, we utilize the holomorphic vector bundle structure of the space of the marked strictly convex real projective structures on a closed surface of genus at least 2 , [20, 27], where Monge-Àmpere equation type argument is used. The method is initiated by Cheng and Yau [8, 9].

\section{HitCHIN MAP AND BUNDLE STRUCTURE}

In this section we collect known results to introduce the Hitchin map. See [20, 22] for details. If $\rho$ is a reductive representation from $\pi_{1}(S)$ to a semisimple Lie group $G$, Corlette proved [11] the following claim: There exists a unique, up to $G, \rho$-equivariant harmonic map $f_{\rho, J}: \tilde{S} \rightarrow G / K$ where $G / K$ is the symmetric space of $G$ and $J$ is a complex structure on $S$. If $f$ is a $\rho$-equivariant map from the universal cover $\tilde{S}$ to $G / K$, then one can define the energy $E(J, f)$ of $f$ with respect to a complex structure $J$ on $S$. Hence given $\rho$, one can define an energy functional $e_{\rho}(J)$ on Teichmüller space by the infimum of energy of $\rho$-equivariant functions with respect to a complex structure $J$. This harmonic map minimizes the energy, i.e. $E\left(J, f_{\rho, J}\right)=e_{\rho}(J)$. The minimum area of $\rho$ is defined to be $\inf _{J} e_{\rho}(J)$. Then it is known [28, 29] that a harmonic map realizing the minimum area of $\rho$ is conformal.

For a map $f$ from $S$ to a Riemannian manifold $(M, g), T f$ can be viewed as a 1 -form on $S$ with values in $f^{*} T M$. Let $T_{\mathbb{C}} f(u)=T f(u)-i T f(J u)$ be a complexified tangent map. 
Then $\mathrm{f}$ is harmonic if and only if $T_{\mathbb{C}} f$ is holomorphic. Furthermore $g_{\mathbb{C}}\left(T_{\mathbb{C}} f, T_{\mathbb{C}} f\right)=0$ if and only if $f$ is minimal.

Every $G$-invariant symmetric multilinear form $P$ on $\mathfrak{g}$ gives rise to a parallel polynomial function $P$, with the same notation, on $G / K$. Hence for any complex structure $J$ on $S$, and for every symmetric $G$-invariant multilinear form $P$ of degree $k$ on $\mathfrak{g}$, any reductive representation $\rho$ gives rise to an element in $\mathcal{Q}(k, J)$ by $P\left(T_{\mathbb{C}} f, \cdots, T_{\mathbb{C}} f\right)$ where $f$ is a $\rho$ equivariant harmonic map. Here $\mathcal{Q}(k, J)=H^{0}\left((S, J), \mathcal{K}^{k}\right)$ is the space of holomorphic $k$-differentials. Denote this map by $F_{P, J}(\rho)=P\left(T_{\mathbb{C}} f, \cdots, T_{\mathbb{C}} f\right)$.

For $G=S L(n, \mathbb{R})$, we can use the symmetric polynomial $P_{k}$ of degree $k$. Then $F_{P_{2}}$ is a metric on $S L(n, \mathbb{R}) / S O(n)$. Set

$$
\Psi_{J}=\bigoplus_{k=2}^{k=n} F_{P_{k}, J} .
$$

Hitchin proved that the map $\Psi_{J}$ is a homeomorphism from the Hitchin component to $\mathcal{Q}(2, J) \oplus \cdots \oplus Q(n, J)$. Set $\epsilon^{(n)}$ to be the bundle over Teichmüller space with fibres $\mathcal{Q}(k, J), k \geq 3$. Labourie introduced the Hitchin map from $\epsilon^{(n)}$ to the Hitchin component

$$
H(J, \omega)=\Psi_{J}^{-1}(\omega)
$$

for $\omega \in \bigoplus_{k=3}^{n} Q(3, J) \oplus \cdots \oplus Q(n, J)$. He showed that

Theorem 3.1. The Hitchin map is surjective.

For $n=3$, this Hitchin map is injective also. Let $\mathcal{T}$ be the Teichmüller space of complex structures $\Sigma_{t}$ on the surface $S$ with the holomorphic tangent space given by $H^{(0,1)}\left(\Sigma_{t}, \mathcal{K}^{-1}\right)$ at each $t \in \mathcal{T}$. Following [20] we consider the space

$$
\mathcal{V}=\left\{(v, t) ; v \in V_{t}:=H^{0}\left(\mathcal{K}_{t}^{3}\right), t \in \mathcal{T}\right\}
$$

Labourie [20] showed that

Theorem 3.2. There exists a mapping class group equivariant diffeomorphism between the moduli space of convex structures on $S$ and the moduli space of pairs $(J, Q)$ where $J$ is a complex structure on $S$ and $Q$ is a cubic holomorphic differential on $S$ with respect to J, i.e. diffeomorphic to $\mathcal{V}$.

Hence the moduli space of convex projective structures can be treated as the holomorphic vector bundle $\mathcal{V}$ over $\mathcal{T}$.

The vector bundle $\mathcal{V}$ (sometimes called Hodge bundle) can be treated as in [4]. Consider the tautological bundle [1]

$$
\hat{T}=\left\{(t, w) ; t \in \mathcal{T}, w \in \Sigma_{t}\right\}
$$

over the Techmüller space $\mathcal{T}$ equipped with the canonical complex structure; see [1, §5]. Note that $\hat{T}$ is then a Kähler manifold with the Kähler metric being locally the product metric. 
The Hodge vector bundle $\mathcal{V}$ is then a complex vector bundle and in particular it is a complex manifold, see e.g. [4]. We let $\mathcal{V}^{*}$ be the dual bundle of $\mathcal{V}$. This can be realized as

$$
\mathcal{V}^{*}=\left\{(v, t) ; v \in V_{t}:=H^{0,1}\left(\mathcal{K}_{t}^{-2}\right), t \in \mathcal{T}\right\}
$$

via the natural mapping class group invariant paring $(f, g)=\int_{\Sigma_{t}} g(f), f \in H^{0,1}\left(\mathcal{K}_{t}^{-2}\right), g \in$ $H^{0}\left(\mathcal{K}_{t}^{3}\right)=H^{1}\left(\mathcal{K}_{t}^{2}\right)$

\section{VARIOUS NOTIONS OF CURVATURE POSITIVITY}

We shall need some results on the positivity for the curvature of the Hodge bundle above. Recall that generally a complex manifold $M$ equipped with a Hermitian metric is said to have a nonpositive bisectional curvature if

$$
R(X, Y, \bar{X}, \bar{Y}) \leq 0
$$

for all $X, Y \in T M \otimes \mathbb{C}$ where $R$ is the curvature tensor extended complex linearly to complexified bundle. When we deal with the holomorphic vector bundles, there are similar notions of positivity (negativity). Let $E$ be a holomorphic vector bundle over a Kähler manifold $M$ and $h$ a Hermitian metric on $E$. Let $\nabla$ be a Chern connection which is compatible with the metric $h$ and complex structure on $E$. If we write $\nabla=\mathcal{D}+\bar{\partial}$, then its curvature $F$ is equal to $\mathcal{D} \bar{\partial}-\bar{\partial} \mathcal{D}$ when acting on local holomorphic sections. More concretely, for any section $s$ and (complexified) vector fields $X, Y$,

$$
F(X, Y)(s)=\nabla_{X} \nabla_{Y} s-\nabla_{Y} \nabla_{X} s-\nabla_{[X, Y]} s .
$$

$F$ is of type $(1,1)$, real and satisfies

$$
h\left(F\left(s_{1}\right), s_{2}\right)+h\left(s_{1}, F\left(s_{2}\right)\right)=0
$$

for any sections $s_{1}$ and $s_{2}$.

If $z^{i}$ are local holomorphic coordinates on $M$ and $e_{\alpha}$ is a local orthogonal frame on $E$, then the curvature $F$ can be written by

$$
\sqrt{-1} F=\sum c_{j \bar{k}}^{\bar{\alpha} \beta} d z^{j} d \bar{z}^{k} \otimes e_{\alpha}^{*} \otimes e_{\beta}
$$

where $\overline{c_{j \bar{k}}^{\bar{\alpha}}}=c_{k j}^{\bar{\beta} \alpha}$. This curvature gives rise to a Hermitian (sesqui-linear) form $\Theta$ on $T M \otimes E$, given locally by

$$
\Theta=\sum c_{j \bar{k}}^{\bar{\alpha} \beta}\left(d z^{j} \otimes e_{\alpha}^{*}\right) \otimes \overline{\left(d z^{k} \otimes e_{\beta}^{*}\right)} .
$$

In tensorial notation, let $e_{\alpha}$ be a local holomorphic frame of $E$ and $e^{\alpha}$ the dual frame, then the curvature tensor $R \in \Gamma\left(M, \wedge^{2} T^{*} M \otimes E^{*} \otimes E\right)$ of $\nabla$ has the form

$$
R=\frac{\sqrt{-1}}{2 \pi} \sum R_{i \bar{j} \alpha}^{\gamma} d z^{i} d \bar{z}^{j} \otimes e^{\alpha} \otimes e_{\gamma}
$$

where $R_{i \bar{j} \alpha}^{\gamma}=h^{\gamma \bar{\beta}} R_{i \bar{j} \alpha \bar{\beta}}$ and

$$
R_{i \bar{j} \alpha \bar{\beta}}=-\frac{\partial^{2} h_{\alpha \bar{\beta}}}{\partial z^{i} \partial \bar{z}^{j}}+\sum h^{\gamma \bar{\delta}} \frac{\partial h_{\alpha \bar{\delta}}}{\partial z^{i}} \frac{\partial h_{\gamma \bar{\beta}}}{\partial \bar{z}^{j}} .
$$


Then the Hermitian vector bundle $(E, h)$ is said to be

(1) Griffith positive if for any nonzero vectors $u=\sum u^{i} \frac{\partial}{\partial z^{i}}$ and $v=\sum v^{\alpha} e_{\alpha}$,

$$
\sum R_{i \bar{j} \alpha \bar{\beta}} u^{i} \bar{u}^{j} v^{\alpha} \bar{v}^{\beta}>0
$$

i.e.,

$$
\Theta(u \otimes v, u \otimes v)=h(F(u, \bar{u})(v), v)>0
$$

for any section $v \neq 0$ and non-zero holomorphic tangent vector field $u$. In other words, $\Theta$ is positive definite on nonzero simple tensors of the form $u \otimes v$.

(2) Nakano positive if for any nonzero vector $u=\sum u^{i \alpha} \frac{\partial}{\partial z^{i}} \otimes e_{\alpha}$,

$$
\sum R_{i \bar{j} \alpha \bar{\beta}} u^{i \alpha} \bar{u}^{j \beta}>0
$$

i.e., the associated sesqui-linear form $\Theta$ is a positive definite Hermitian form.

(3) dual Nakano positive if for any nonzero vector $u=\sum u^{i \alpha} \frac{\partial}{\partial z^{i}} \otimes e_{\alpha}$,

$$
\sum R_{i \bar{j} \alpha \bar{\beta}} u^{i \beta} \bar{u}^{j \alpha}>0 .
$$

It is obvious from the definition that Nakano positivity implies Griffith positivity. Also $(E, h)$ is dual Nakano positive if and only if $\left(E^{*}, h^{*}\right)$ is Nakano negative. If $E$ is Griffith positive then its dual $E^{*}$ is Griffith negative. We also remark that Griffith positivity implies that the bundle $E$ is ample.

In particular if $E$ is a line bundle, then the above notions of positivity all agree. The curvature in this case is computed by $\bar{\partial} \partial \log \|w\|^{2}$ for any local holomorphic frame (i.e. non zero-section) $w$. We will use these notions of positivity to prove the existence of Kähler metric on the Hitchin component of $\chi\left(\pi_{1}(S), S L(3, \mathbb{R})\right)$.

\section{KÄHLER PROPERTY}

The following result is a corollary of a general theorem of Berndtsson [4] applied to the vector bundle $\mathcal{V}$; when we replace the fiber of the bundle $\mathcal{V}$ by the spaces $H^{0}\left(\mathcal{K}_{t}^{2}\right)$ viewed as the dual space of the holomorphic tangent space of Teichmüller space, it is a classical result of Ahlfors [2] that the Teichmüller space has negative holomorphic sectional curvature equipped with the Weil-Petersson metric.

Theorem 5.1. The bundle $\mathcal{V}$ is Griffith positive.

Proof. The tangent line bundle $\mathcal{K}_{t}^{-1}$ on each Riemann surface $\Sigma_{t}$ is equipped with a unique Kähler-Einstein metric of negative curvature -1 , in other words, the canonical line bundle $\mathcal{K}_{t}$ is Kähler-Einstein of positive curvature 1 . Let $\mathcal{L}$ on $\hat{T}$ be the pull-back of the line bundle $\mathcal{K}_{t}$ to $\hat{T}$ under the projection $\hat{T} \rightarrow \mathcal{T}$. It follows from [32, Theorem 5.5, Lemma 5.8] that the bundle $\mathcal{L}$ is positive; see also [30, Main Theorem, Theorem 1] for generalization. Thus $\mathcal{L}^{2}$ is positive since $\mathcal{L}$ is a line bundle. In [4], it is proved that the Hermitian vector bundle over $\mathcal{T}$,

$$
H^{0}\left(\Sigma_{t}, L_{0} \otimes \mathcal{K}_{\hat{T} / \mathcal{T}}\right) \mapsto t
$$


the fiber being the spaces of global sections, endowed with $L^{2}$-metric, is Nakano positive, where $L_{0}$ is any positive line bundle over the Kähler manifold $\hat{T}$. In particular taking $L_{0}=\mathcal{L}^{2}$ we have

$$
H^{0}\left(\Sigma_{t}, \mathcal{L}^{2} \otimes \mathcal{K}_{\hat{T} / \mathcal{T}}\right)=H^{0}\left(\Sigma_{t}, \mathcal{K}_{t}^{2} \otimes \mathcal{K}_{\hat{T} / \mathcal{T}}\right)=H^{0}\left(\Sigma_{t}, \mathcal{K}_{t}^{3}\right)
$$

we see that the bundle with fiber $H^{0}\left(\mathcal{K}_{t}^{3}\right)$ over $\mathcal{T}$, i.e., the bundle $\mathcal{V}$ is Nakano positive, hence Griffith positive.

Remark 5.2. The Nakano positivity of the bundle $H^{0}\left(\Sigma_{t}, \mathcal{K}_{t}^{3}\right)$ can also be directly proved by using the result of Berndtsson in [5]. It is proved there whenever the metric on $L_{0}$ is positive fiberwise then the Nakano positivity still holds for $H^{0}\left(\Sigma_{t}, L_{0} \otimes \mathcal{K}_{\hat{T} / \mathcal{T}}\right)$. In our case the fiber metric is dual to the hyperbolic metric on the Riemann surfaces and has constant positive curvature. See also [25, 26] for related works.

Corollary 5.3. The bundle $\mathcal{V}^{*}$ is Griffith negative.

The first statement below can be proved for general bundles with the Griffith negativity, and here we are only interested in the special case of $\mathcal{V}^{*}$.

Theorem 5.4. The bundle $\mathcal{V}^{*}$ is a Kähler manifold. In particular the Hitchin component of the character variety $\chi\left(\pi_{1}(S), S L(3, \mathbb{R})\right)$ has a mapping class group invariant Kähler metric.

Proof. Let $w_{0} \neq 0$ be a fixed point in $\mathcal{V}^{*}$ with $z_{0}=\pi\left(w_{0}\right)$, where $\pi: \mathcal{V}^{*} \rightarrow \mathcal{T}$ is the defining projection. We choose a local trivializing holomorphic frame $\left\{e_{\alpha}=e_{\alpha}(z)\right\}$ in a coordinate neighborhood $U$ of $z_{0}$, and write, with some abuse of notation, $z=$ $\left(z^{1}, \cdots, z^{n}\right)$ as the coordinate of $U$. The local holomorphic coordinates near $w_{0}$ will be $w=\sum_{\alpha} x^{\alpha} e_{\alpha}(z) \rightarrow\left(z^{i}, x^{\alpha}\right)$, and the holomorphic tangent vectors are $T=(u, v)$ with $u=\sum u_{i} \frac{\partial}{\partial z^{i}}$ and $v=\sum v_{\alpha} \frac{\partial}{\partial x^{\alpha}}$.

We let $\psi$ be a local Kähler potential for $\mathcal{T}$ near $z_{0}, \mathcal{T}$ being equipped with the WeilPetersson metric. Thus $\pi^{*} \psi(w)$ is defined in a neighborhood of $w_{0}$. We let

$$
\phi(w)=\|w\|^{2}+\psi(\pi(w))=\|w\|^{2}+\pi^{*} \psi(w)
$$

to be defined in a neighborhood of $w_{0}$.

Let $\nabla=\mathcal{D}+\bar{\partial}$ be the Chern connection acting on sections of $\mathcal{V}^{*}$ and $\mathcal{D} e_{\alpha}=\sum \theta_{\alpha}^{\beta} e_{\beta}$ where $\theta_{\alpha}^{\beta}$ is type $(1,0)$. We fix $T=(u, v) \neq 0$ at $w_{0}$ and perform the differentiation $\bar{\partial}_{T} \partial_{T} \phi$ with $\partial_{T}=\partial_{u}+\partial_{v}$. The vectors $w=\sum_{\alpha} x^{\alpha} e_{\alpha}(z)$ will be viewed as holomorphic sections for fixed $x_{\alpha}$. The curvature $R(u, \bar{u})$ of Chern connection $\nabla$ is

$$
R(u, \bar{u}) w=\nabla_{u} \nabla_{\bar{u}} w-\nabla_{\bar{u}} \nabla_{u} w-\nabla_{[u, \bar{u}]} w=\nabla_{u} \bar{\partial}_{u} w-\bar{\partial}_{u} \mathcal{D}_{u} w=-\bar{\partial}_{u} \mathcal{D}_{u} w
$$

using $\bar{\partial}_{u} w=0$ and $[u, \bar{u}]=0$. We have

$$
\begin{gathered}
\partial_{u}\|w\|^{2}=\left(\mathcal{D}_{u} w, w\right)+\left(w, \bar{\partial}_{u} w\right)=\left(\mathcal{D}_{u} w, w\right), \\
\bar{\partial}_{u} \partial_{u}\|w\|^{2}=\bar{\partial}_{u}\left(\mathcal{D}_{u} w, w\right)=\left(\bar{\partial}_{u} \mathcal{D}_{u} w, w\right)+\left(\mathcal{D}_{u} w, \mathcal{D}_{u} w\right)=-(R(u, \bar{u}) w, w)+\left(\mathcal{D}_{u} w, \mathcal{D}_{u} w\right) .
\end{gathered}
$$


Since

$$
\mathcal{D}_{u} w=\sum D_{u} x^{\alpha} e_{\alpha}+\sum x^{\alpha} \mathcal{D}_{u} e_{\alpha}=\sum x^{\alpha} \theta_{\alpha}^{\beta}(u) e_{\beta}(z)
$$

$\theta_{\alpha}^{\beta}(u) e_{\beta}(z)$ depends smoothly only on $z$, and hence $\bar{\partial}_{v} \mathcal{D}_{u} w=0$. Also $\partial_{v} w=v$, hence

$$
\bar{\partial}_{v} \partial_{u}\|w\|^{2}=\left(\mathcal{D}_{u} w, v\right), \quad \bar{\partial}_{u} \partial_{v}\|w\|^{2}=\left(v, \mathcal{D}_{u} w\right)
$$

and

$$
\bar{\partial}_{v} \partial_{v}\|w\|^{2}=(v, v)
$$

Thus

$$
\begin{aligned}
\bar{\partial}_{T} \partial_{T}\|w\|^{2}=-(R(u, \bar{u}) w, w) & +\left(\mathcal{D}_{u} w, \mathcal{D}_{u} w\right)+\left(\mathcal{D}_{u} w, v\right)+\left(v, \mathcal{D}_{u} w\right)+(v, v) \\
& =-(R(u, \bar{u}) w, w)+\left(\mathcal{D}_{u} w+v, \mathcal{D}_{u} w+v\right) \geq 0
\end{aligned}
$$

Here we use Griffith negativity of $\mathcal{V}^{*}$ to have $(R(u, \bar{u}) w, w)<0$. We have also

$$
\bar{\partial}_{T} \partial_{T} \pi^{*} \psi=\bar{\partial}_{u} \partial_{u} \psi
$$

which is positive definite in $u$. Thus

$$
\bar{\partial}_{T} \partial_{T} \phi=-(R(u, \bar{u}) w, w)+\left(\mathcal{D}_{u} w+v, \mathcal{D}_{u} w+v\right)+\bar{\partial}_{u} \partial_{u} \psi=I+I I+I I I,
$$

a sum of two semi-positive Hermitian forms. We prove that it is positive at $w=w_{0}$. Suppose the quadratic form vanishes. Then $I=I I=I I I=0$. If $w_{0} \neq 0$, then $I=0$ implies that $u=0$ by Griffith negativity, and the second term is $0=I I=(v, v)$, which implies further that $v=0$. Suppose $w_{0}=0$, i.e., in the zero section. Then $I I I=0$ implies that $u=0$, which in turn implies $0=I I=(v, v)$ and $v=0$. In either cases, $T=(0,0)$, a contradiction to the choice of $T=(u, v) \neq 0$.

It follows from the definition that the map $w \rightarrow-w$ is an isometry and its fixed point set is the space of zero sections, namely the Teichmüller space identified as a submanifold. We have thus

Corollary 5.5. Let Teichmüller space $\mathcal{T}$ be equipped with the mapping class group invariant Weil-Petersson metric and the Hitchin component $\chi_{H}\left(\pi_{1}(S), S L(3, \mathbb{R})\right)$ of the character variety $\chi\left(\pi_{1}(S), S L(3, \mathbb{R})\right)$ be equipped with the mapping class group invariant Kähler metric as in Theorem 5.4 Then $\mathcal{T}$ is a totally geodesic submanifold of $\chi_{H}\left(\pi_{1}(S), S L(3, \mathbb{R})\right)$.

Recenlty Labourie [23] generalized this theorem to the Hitchin components associated to all real split simple Lie groups of rank 2.

Remark 5.6. If we consider the bundle $\mathcal{W}$ over Teichmüller space whose fiber over $t$ equal to $\sum_{j \geq 2} H^{0}\left(\Sigma_{t}, \mathcal{K}_{t}^{j}\right)$, then it is Griffith positive and the same method applies to show that the total space $\mathcal{W}^{*}$ has a mapping class group invariant Kähler metric. For example, the space of the marked complex projective structures on a closed surface of genus at least 2 is a holomorphic vector bundle over Teichmüller space with fibers being 
the space of holomorphic quadratic differentials. Hence this space has a natural mapping class group invariant Kähler metric.

\section{REFERENCES}

[1] L. V. Ahlfors, Some remarks on Teichmüller's space of Riemann surfaces, Ann. of Math. 74 (2) (1961), 171-191, MR0204641, Zbl 0146.30602.

[2] L. V. Ahlfors, Curvature properties of Teichmüller's space, J. Analyse Math. 9 (1961/1962), 161-176, MR0136730, Zbl 0148.31201.

[3] Y. Benoist and D. Hulin, Cubic differentials and finite volume convex projective surfaces, Geom. Topol. 17 (2013), 595-620, MR3039771, Zbl 1266.30030.

[4] B. Berndtsson, Curvature of vector bundles associated to holomorphic fibrations, Ann. of Math. (2), 169 (2009), 531-560, MR2480611, Zb1 1195.32012.

[5] B. Berndtsson, Strict and nonstrict positivity of direct image bundles, Math. Z., 269 (2011), 12011218, MR2860284, Zbl 1252.32027.

[6] M. Bridgeman, R. Canary, F. Labourie, and A. Sambarino, The pressure metric for convex representations, GAFA. 2 (2015), 1089-1179, MR3385630, Zbl 06483696.

[7] S. Choi and W. Goldman, Convex real projective structures on closed surfaces are closed, Proc. A.M.S. 118 (1993), 657-661, MR1145415, Zbl 0810.57005.

[8] S. Cheng and S. Yau, On the regularity of the monge-ampére equation $\operatorname{det}\left(\partial^{2} u / \partial x_{i} \partial s x_{u}\right)=F(x, u)$, Comm. Pure Appl. Math., 30 (1977), 41-68, MR0437805, Zbl 0347.35019.

[9] S. Cheng and S. Yau, Complete affine hypersurfaces. I. The completeness of affine metrics, Comm. Pure Appl. Math. 39 (1986), 839-866, MR0859275, Zbl 0623.53002.

[10] D. Cooper, D. Long and S. Tillmann, On convex projective manifolds and cusps, Adv. Math. 277 (2015), 181-251, MR3336086, Zbl 06431971.

[11] K. Corlette, Flat G-bundles with canonical metrics, J. Diff. Geom. 28 (1988), no. 3, 361-382, MR0965220, Zbl 0676.58007.

[12] M. Crampon, Entropies of strictly convex projective manifolds, J. Mod. Dyn. 3 (2009), 511-547, MR2587084, Zbl 1189.37034.

[13] W. Goldman, The symplectic geometry of affine connections on surfaces, J. Reine Angew. Math. 407 (1990), 126-159, MR1048531, Zbl 0692.53015.

[14] W. Goldman, Convex real projective structures on compact surfaces, J. Diff. Geom, 31 (1990), 791845, MR1053346, Zbl 0711.53033.

[15] N. Hitchin, The self-duality equations on a Riemann surface, Proc. London Math. Soc. (3) 55 (1987), 59-126, MR0887284, Zbl 0634.53045.

[16] N. Hitchin, Lie groups and Teichmüller space, Topology, 31(1992), 449-473, MR1174252, Zbl 0769.32008.

[17] I. Kim, Rigidity and deformation spaces of strictly convex real projective structures on compact manifolds, J. Diff. Geom. 58 (2001), 189-218, MR1913941, Zbl 1076.53053. Erratum, J. Diff. Geom. 86 (2010), 189, MR2772549, Zbl 1213.53057.

[18] I. Kim, Compactification of strictly convex real projective structures, Geom. Dedicata 113 (2005), 185-195, MR2171304, Zbl 1087.51008.

[19] I. Kim and G. Zhang, Eichler-Shimura isomorphism for complex hyperbolic lattices, submitted.

[20] F. Labourie, Flat projective structures on surfaces and cubic holomorphic differentials, Pure Appl. Math. Q, 3 (2007), 1057-1099, MR2402597, Zbl 1158.32006.

[21] F. Labourie, Anosov flows, surface groups and curves in projective space, Invent. Math. 165 (2006), 51-114, MR2221137, Zbl 1103.32007.

[22] F. Labourie, Cross ratios, Anosov representations and the energy functional on Teichmüller space, Ann. Sci. E.N.S. 41 (2008),437-469, MR2482204, Zbl 1160.37021.

[23] F. Labourie, Cyclic surfaces and Hitchin components in rank 2, preprint available at http://arxiv.org/pdf/1406.4637.pdf.

[24] Q. Li, Teichmüller space is totally geodesic in Goldman space, preprint. 
[25] K. Liu, X. Sun and S. Yau, Canonical metrics on the moduli space of Riemann surfaces. I., J. Diff. Geom. 68 (2004), 571-637, MR2144543, Zbl 1078.30038.

[26] K. Liu, X. Sun and S. Yau, Canonical metrics on the moduli space of Riemann surfaces. II., J. Diff. Geom. 69 (2005), 163-216, MR2169586, Zbl 1086.32011.

[27] J. Loftin, Affine spheres and convex $R P^{n}$ manifolds, American Journal of Math. 123 (2) (2001), 255-274, MR1828223, Zbl 0997.53010.

[28] J. Sacks and K. Uhlenbeck, Minimal immersions of closed Riemann surface, Trans. AMS 271 (1982), no. 2, 639-652, MR0654854, Zbl 0527.58008.

[29] R. Schoen and S. Yau, Existence of incompressible minimal surfaces and the topology of threedimensional manifolds with nonnegative scalar curvature, Ann. of Math. (2) 110 (1979), no. 1, 127142, MR0541332, Zbl 0431.53051.

[30] G. Schumacher, Positivity of relative canonical bundles and applications, Invent. Math. 190 (2012), 1-56, MR2969273, Zbl 1258.32005.

[31] C. Simpson, Higgs bundles and local systems, Publ. Math. Inst. Hautes Études Sci. 75 (1992), 5-95, MR1179076, Zbl 0814.32003.

[32] S. Wolpert, Chern forms and the Riemann tensor for the moduli space of curves, Invent. Math. 85 (1986), 119-145, MR0842050, Zbl 0595.32031.

School of Mathematics, KiAS, Heogiro 85, Dongdaemun-Gu SeOul, 130-722, RepubliC

OF KOREA. EMAIL: INKANG@KIAS.RE.KR

Mathematical Sciences, Chalmers University of TeChnology And Mathematical SciENCES, GÖteborg University, SE-41296 GöteborG, SWEDEn. EMAIL: GENKAi@CHALMERS.SE 